\title{
Analyzing Cross-Cultural Studies of Arguing in the Globalized World
}

\author{
Iryna Khomenko
}

\author{
Doctor of Philosophical Sciences, Professor, \\ Taras Shevchenko National University of Kyiv (Kyiv, Ukraine) \\ E-mail: khomenkoi.ukr1@gmail.com \\ https://orcid.org/0000-0003-3522-2271
}

Cosmology is often viewed as the study of the physical universe. However, it does not mean that the issues of philosophy of cosmology relate only to research fields of physics, astronomy, astrophysics, and mathematics. It should be noted that cosmology explores the physical situation in the large context of human existence.

In global times, new issues arise in the field of philosophical cosmology. How is the human mind changing with the improvement of human knowledge about the universe? What knowledge and skills should have a person of the future? Is it possible to create a universal human community, members of which will explore space, fly and live beyond the Earth? Can people of various cultures communicate with each other within this community?

For the answers, scholars should conduct theoretical as well as empirical research. In this context, I have turned to current cross-cultural studies of communication, in which researchers explore how people from various cultural perspectives establish a dialogue in global times and what challenges they face. In the paper, I focus on the international project of comparing arguing in various countries across the globe. In my mind, its findings are important for two reasons: a substantial and a methodological one. I pay particular attention to the latter.

Keywords: philosophy of cosmology, globalized world, person of the future, cross-cultural studies, face to face arguing, empirical research

Received: July 18, 2019; accepted: August 29, 2019

Philosophy and Cosmology, Volume 24, 2020: 91-98.

https://doi.org/10.29202/phil-cosm/24/9

\section{Introduction}

The term cosmology is often viewed as the study of the physical universe. However, it does not mean that the issues of philosophy of cosmology relate only to research fields of physics, astronomy, astrophysics, and mathematics. In this regard, it should be noted that one of the key features of cosmology is to explore "the physical situation that is the context in the large for human existence: the universe has such a nature that our life is possible. This means that although it is a physical science, it is of particular importance in terms of its

(C) Khomenko, Iryna, 2020 
implications for human life" (Smeenk \& Ellis, 2017). It is no coincidence that most religions and cultures include some cosmology treatments due to explain the universe as totally and its conceptual foundations. In global times, the new issues arise in the field of philosophy of cosmology. Among them are the following. How is the human mind changing with the improvement of human knowledge about the universe? What knowledge and skills should have a person of the future? Is it possible to create a universal human community, members of which will explore space, fly and live beyond the Earth? Can people of various cultures communicate with each other within this community?

For the answers to these issues, it is necessary to carry out theoretical as well as empirical research. In this context, I would like to turn to current cross-cultural studies of communication, in which researchers explore how people from various cultural perspectives establish a dialogue in global times and what challenges they face. In the paper, I focus on the international project of comparing arguing in various countries across the globe. In my mind, its findings are important for two reasons: a substantial and a methodological one.

In the first case, scholars investigate how various cultural features (for example high/ low reverence for authority, subjectivism/collectivism, high/low avoidance of uncertainty, more/less confrontational style of arguing, extrovert/introvert, more/less verbose style of communication, etc.) influence the argumentation in daily encounters. To date, the United States, Chile, China, France, India, Malaysia, Mexico, the Netherlands, Portugal, South Korea, Turkey, Ukraine, and United Arab Emirates have already participated in this global project.

The following point could be emphasized about the second reason. Taking into account that cross-cultural studies of argumentation are significantly important in global times, the relevant task is to develop the methodology of such empirical research and to create instruments, which scholars may use.

In this paper, I would like to explore the key characteristics of the methodology of crosscultural studies of arguing and to justify that such tool as survey widely used in the fields of sociology and psychology can be brought in to fill the gap in the methodology of the empirical studies in argumentation field.

\section{Empiricalization as one the main prospects of argumentation research}

It should be noted that it is a problem for the modern theory of argumentation that often it is presented only as numerous theoretical models based on various grounds of reasonableness. For building such models, scholars have elaborated many useful tools. However, the argumentation study cannot be reduced only to the theoretical area. I believe most scholars would agree that nowadays in the field of argumentation empirical investigations are increasingly important.

Let us look at the history of this issue. In 1958 Stephen Toulmin, the founder of the working logic, in his epoch-making book The Uses of Argument proposed a procedural model of argumentation (Toulmin, 1958). He emphasized "a radical re-ordering of logical theory is needed in order to bring it more nearly into the line with critical practice" (Toulmin, 1958: 253; Toulmin, 2003: 234). It means that his model could work adequately in the different areas of argumentative reality.

In this regard, Stephen Toulmin assumed that not only a theoretical component is relevant to the study of argumentation but also an empirical one is needed. Justifying this view he claimed: "logic ...may have to become less of an a priori subject than it has 
recently been... Accepting the need to begin by collecting for study the actual forms of argument current in any field, our starting point will be confessedly empirical" (Toulmin, 1958: 257; Toulmin, 2003: 236-238). In addition, it should be stressed that Stephen Toulmin connected the empirical component with the historical one. He believes that "not only will logic have to become more empirical; it will inevitably tend to be more historical... In the natural science, for instance, men such as Kepler, Newton, Lavoisier, Darvin and Freud have transformed not only our beliefs but also our ways of arguing and our standards of relevance and proof... Grotius and Bentham, Euclid and Gauss, have performed the same double feat for us in other fields" (Toulmin, 1958: 257; Toulmin, 2003: 237). In fact, here Stephen Toulmin bearded in mind that empirical database for scholars may be the history of thought in general and the history of science in particular.

Chaim Perelman and Lucy Olbrechts-Tyteca, who are among the co-founder of the modern theory of argumentation, supported Toulmin's view. They claimed that the theoretical concepts of their treatment, called new rhetoric, had to base on the empirical observation (Perelman \& Olbrechts-Tyteca, 1958). Unfortunately, the empirical dimension in their treatment was not developed thoroughly.

Thus, it may be maintained that these scholars in their treatments widened substantially the vision on the empirical component in argumentation studies. However, it is disappointing to note that after that for a long time research in this field was still mainly aimed at theoretical proposals and their philosophical support. Only more recently empiricalization has become a new trend among argumentation scholars. For example, Frans van Eemeren considers empiricalization as one of the main prospects of current argumentation research (Eemeren, 2015, 2017). In his opinion, "three major developments in the treatment of argumentation have begun to materialize that open new avenues for research. Although they differ in shape, these developments can be observed across a broad spectrum of theoretical approaches. The three developments I have in mind can be designated as empiricalization, contextualization, and formalization of the treatment of argumentation" (Eemeren, 2015: 5).

Due to the increasing importance of empiricalization, a set of questions arise. Among them are the following. What does empiricalization involve? Could we speak about empirical methodology in the field of argumentation? What do mean the notions: empirical analysis, empirical research, empirical approach, empirical evidence, and empirical method in the study of argumentation?

It should be noted that a key feature of empirical research is to use empirical evidence as a way of gaining results. The term empirical evidence usually refers to the systematic collection and analysis of data related to the field of argumentation. Scholars may involve a variety of instruments to these ends (Khomenko, 2018).

As mentioned above Stephen Toulmin meant by the term empirical that his argumentation model could work adequately in the different areas of argumentative reality, particularly in the field of law. Chaim Perelman and Lucy Olbrechts-Tyteca believed that their new rhetoric had to base on such method as an empirical observation.

Many different methods are currently being pursued, allowing for flexibility, when addressing the needs of researchers. In this regard, it could be pointed out on some empirical approaches in the field of argumentation. Among them is historical-textual and the metaargumentation approaches elaborated by Moris Finocchiaro. He has produced a number of highly regarded works, in which the scholar has revealed the key features of his approaches and showed some applications of own methodological views (Finocchiaro, 1980, 1994, 2005, 2010, 2013). 
In empirical investigations of various historical texts, Moris Finocchiaro used the method of alternative conclusion, active evaluation, ad hominem argument, the method of counterexample, the principle of charity, and explanation of the error in reasoning. In his mind, the first three methods are the most relevant for his historical-textual approach of argumentation (Finocchiaro, 1980; 1994; 2005; 2010). Besides, Moris Finocchiaro elaborated the method of meta-argumentation based on the principle of interpretation and evaluation (Finocchiaro, 2013).

In addition, it should be mentioned the cross-cultural approach originated in the US. Dale Hample contributed significantly to drafting a methodological basis for this project (Hample, 2003; 2005; 2018). Dale Hample and other argumentation scholars from various countries have widely explored orientations and understandings of interpersonal arguing. Here scholars in order to collect data often use a survey as an instrument of such investigations (Hample, 2003; 2005; 2018).

Taking into account mentioned above, it could be argued that the term empirical evidence is currently used in two ways. In the narrower context, this term is viewed as information or data acquired by observation, experimentation or survey. However, such empirical evidence in itself is not a great value. It can be identified only as material for further investigations.

In the case of empirical evidence in the broader context should bear in mind that here the term evidence can be used as a proof of argumentation scholar's standpoint or hypotheses. In this regard, I believe it is interesting to refer to Kant's terminology from his Critique of Pure Reason (Kant, 1999).

According to this, theoretical justification, particularly logical proof should be based on a priori analysis. Here it is possible to argue some claim without using empirical methods. Such evidence is based purely on applying various types, forms or schemes of reasoning. As a rule, it relates to some procedures, which are based on certain rules and laws.

In contrast, empirical evidence relies on a posteriorly analysis. It involves that (1) something should be observed, measured, calculated, or tested; (2) all empirical information and data could be analyzed quantitatively as well as qualitatively. Many argumentation scholars combine both forms of analysis to better answer to questions, which cannot be studied in artificial laboratory settings. Conclusions drawn in this way help to give answers to research questions of a certain project. In my mind, term empirical evidence could be especially interesting for argumentation scholars in this meaning.

Let us consider carefully what is empirical evidence in cross-cultural studies in the field of argumentation.

\section{Empirical evidence in cross-cultural studies of face-to-face arguing}

Nowadays many methodological approaches can be reasonably applied to the goal of understanding how people with different cultural traditions relate to face-to-face arguing. Among the appropriate approaches is the project selected here. Its orientation is to examine predispositions and understandings regarding interpersonal arguing. Its main objective is to collect and analyze the data summarizing fundamental orientations to arguing among respondents. All of the instruments of this research originated in the US. It should be stressed that besides elucidating the argumentation predispositions in a certain country this approach advances the general project of comparing argumentation in various countries across the globe (Hample, 2018). To date, a lot of countries have already participated in this global project (Hample \& Anagondahalli, 2015; Hample \& Rapanta, 2015; Lewiński et al., 2018; Khomenko \& Hample, 2019). This fact allows comparing and generalizing the 
data collected in different countries and regions. In 2018, Ukraine jointed this international project. It was the first comprehensive attempt to understand and assess the sentiments of Ukrainians towards face-to-face arguing. The main objective of the cross-cultural project was to collect and analyse data summarizing fundamental orientations to arguing among Ukrainians.

Let us consider the key questions concerning the methodology of empirical evidence in mentioned above projects.

Q1. In what way can the data be collected within the international cross-cultural project on face-to-face arguing?

A1. The empirical part of such project includes nationwide surveys. Data can be collected relatively quickly, using a standard set of questions. The polls can be carried out via online (for example, see Survey-Monkey.com).

Q2. How are research questions formulated?

A2. The first step in conducting a poll is to formulate the research questions. As examples, we can consider the questions, used in the projects from India, United Arab Emirates, Portugal, and Ukraine.

\section{A. India}

RQ1: To what degree (if any) does sex differences influence argumentation in India?

RQ2: What are the relationships among the constructs (argument motivations, argument frames, conflict personalization) for Indians?

RQ3: To what degree (if any) do Indians differ from Americans in their orientations (means) toward argumentation?

RQ4: To what degree (if any) do Indians differ from Americans in the relationships (correlations) among the argumentation constructs? (Hample \& Anagondahalli, 2015)

\section{B. United Arab Emirates}

RQ1: Does the UAE display the same relationships among variables that describe interpersonal arguing as in other nations?

RQ2: Are the mean scores from the Emirati population comparable to those of other nations? (Hample \& Rapanta, 2015)

C. Portugal

RQ1: Do Portuguese men and women differ in their orientations and understandings of interpersonal arguing, as measured by verbal aggressiveness, argumentativeness, argument frames, and personalization of conflict?

RQ2: Do Portuguese and US respondents have different mean scores on the instruments assessing orientations and understandings of interpersonal arguing?

RQ3: What are the correlational systems among the variables in Portugal, and are they comparable to those in the US? (Lewiński et al., 2018).

\section{Ukraine}

RQ1: How do Ukrainian and US respondents compare in their average responses to items concerning arguing motivations, understandings, and reactions?

RQ2: Do Ukrainian men and women differ in their arguing motivations, understandings, and reactions?

RQ3: Do Ukrainian respondents who chose the Ukrainian language version of the survey differ in their arguing motivations, understandings, and reactions, compared to those who chose the Russian language version?

RQ4: What are the internal associations among arguing motivations, understandings, and reactions for the Ukrainian sample (Khomenko \& Hample, 2019)? 
As can we see although research questions are different in each specific case, they unite around a common goal of the international project - to examine predispositions and understandings regarding interpersonal arguing. Also, it should be noted that some research questions are internal to a certain country, while also several of these compare it to other countries, participated in this cross-cultural project.

Q3. What was the methodology of questionnaire design?

A3. A survey base on the questionnaire, which is a research instrument consisting of a series of questions for the purpose of gathering information from respondents. As mentioned above, this approach is based on the methodology survey used in the overall global project summarized in Hample (2018). The English language versions of the main instruments can be found in Hample (2018), Infante and Rancer (1982), and Infante \& Wigley (1986). Scholars need to translate the origin questionnaire. For example, due to the bilingualism of the majority of the Ukrainian population, two versions of the survey (Ukrainian and Russian) were composed. The participants had a free choice as to which to complete the survey.

The first set of questions in the survey is devoted to ordinary demographics. The sample Respondent represents the participants by gender, age, a region of residence, and student/ employment status.

The second set of questions deal with argument motivations and is connected with concerned argumentativeness (Infante \& Rancer, 1982) and verbal aggressiveness (Infante \& Wigley, 1986). Argumentativeness is the impulse to attack the other arguer's premises, reasons, and evidence. Verbal aggressiveness is considered as the predisposition to engage in ad hominem attacks on the other arguer. It also has two subscales, VA-antisocial and VAprosocial, which need to be analyzed separately rather than being merged into one "verbal aggressiveness" score. These instruments collectively describe people's inclinations to argue about the substance of disagreement and to attack the other arguer personally.

The third set of questions assesses the degree to which people take conflicts personally (TCP). The scales can be found in Hample (2018). The last battery of measures concerns personalization of conflict.

The fourth set of questions is devoted to argument frames, a battery of instrument intends to capture people's understandings of the project of arguing face-to-face (current items are in Hample, 2018). Shortly speaking, argument frames (Hample, 2003, 2005, 2018) is a battery of instruments intended to answer the question: "What do ordinary people think they are doing when they argue?"

Collected together, these measures should give full information about how people in the certain country understand interpersonal arguing, what their goals for arguing are, how they communicate to the other arguer, what their motivations, and what are subjects they are focused on in face to face conflicts.

Q4. Who were the respondents of the nationwide surveys?

A4. Most respondents who participated in the polls in various countries were mostly undergraduates, $\mathrm{PhD}$ students, and staff from different universities. In addition, the members of their families, friends, and neighborhoods were the also poll respondents.

Q5. What is the data analysis in a cross-cultural project?

A5. The obtained data can be analyzed quantitatively and qualitatively. Firstly, based on descriptive statistics on all the measurements scholars (1) fully explore the data and (2) compare the average responses to all items, contained in the various nationwide surveys. It is a quantitative analysis. 
The second step is to explore the findings qualitatively. The goals of such analysis are (1) to generalize all quantitative results, (2) to draw conclusions, (3) to discuss these conclusions.

Let us illustrate mentioned above in the case of Ukraine-the US project (Khomenko \& Hample, 2019). Firstly, based on statistics on all the measurements the project team compared the average responses to all items: (1) mean differences between Ukrainian and US respondents, concerning arguing motivations, understandings, and reactions; (2) mean differences between Ukrainian men and Ukrainian women and between Ukrainian and Russian language choosers; (3) correlations among Argument Motivation Measures (Ukraine), (4) correlations among Argument Frames Measures (Ukraine), (5) correlations among Taking Conflict Personally Measures (Ukraine).

Then the qualitative analysis of the quantitative results allowed the project team to generalize all findings and to draw conclusions. Among them were the following.

First, Ukrainians and US respondents did, actually, differ in their mean responses to the instruments used in the survey. "The standout result was that Ukrainians felt that arguing was a far less civil activity than was the case in the US. This civility finding was paralleled by Ukrainians' beliefs that conflict tends to damage relationships" (Khomenko \& Hample, 2019: 634). Second, we concluded that "Ukrainian men and women were quite distinct in their orientations to the interpersonal argument. Generally, men were more aggressive and women more passive and easily hurt" (Khomenko \& Hample, 2019: 635). Third, our conclusion on the point of the surveys' language, which the respondents choose is that the language made no difference. "To the degree that language preference implies something about national identity, we found no evidence that national identity carried any special predispositions about face-to-face arguing" (Khomenko \& Hample, 2019: 635). Also, exploring the question of the internal associations among arguing motivations, understandings, and reactions for the Ukrainian sample, we came to the conclusion, that the Ukrainian results were generally quite conformable to US results. Motivations had US patterns, as did the argument frames and the TCP measures" (Khomenko \& Hample, 2019: 637).

Besides mentioned above, the qualitative analysis involves a discussion about the obtained conclusions. You can see the discussion in the case of Ukraine-the US project in this paper (Khomenko \& Hample, 2019: 637-639).

\section{Conclusions}

In this paper, I have focused on current cross-cultural studies of communication. Their goal is to explore how people from various cultural perspectives establish a dialogue in global times and what challenges they face. I have considered the international project of comparing arguing in various countries across the globe, particularly its methodology and instruments.

Finally, I have identified its methodology as the methodology of empirical evidence. At the beginning, the main scholar's task is to collect data by conducting nationwide surveys. Then researchers should analyse the database. Based on descriptive statistics on all the measurements scholars (1) fully explore the data and (2) compare the average responses to all items, contained in the various nationwide surveys. It is a quantitative analysis. Then they explore the findings qualitatively. Their goals are (1) to generalize all quantitative results, (2) to draw conclusions, and (3) to discuss them. 


\section{References}

Eemeren, Frans Hendrik van. (2017) Argumentation theory and argumentative practices: A Vital but Complex Relationship. Informal Logic, 37 (4), 322-350, https://doi.org/10.22329/ il.v37i4.5002

Eemeren, Frans. (2015) Hendrik van. Bingo! Promising developments in argumentation theory. In Reflections on theoretical issues in argumentation theory. Heidelberg etc.: Springer, 3-25.

Finocchiaro, Maurice A. (2013) Meta-argumentation. An approach to logic an argumentation theory. London: College Publications.

Finocchiaro, Maurice A. (2010) Defending Copernicus and Galileo. Critical reasoning in the two affairs. Dordrecht: Springer.

Finocchiaro, Maurice. (2005) Arguments about arguments. Systematic, critical and historical essays in logical theory. Cambridge: Cambridge University Press.

Finocchiaro, Maurice A. (1994) Two empirical approaches to the study of reasoning. Informal Logic, 1994, 16: 1-21, https://doi.org/10.22329/il.v16i1.2431

Finocchiaro, Maurice A. (1980) Galileo and the art of reasoning: rhetorical foundations of logic and scientific method, Boston, MA: Reidel.

Hample, Dale. (2018) Interpersonal arguing, New York: Peter Lang.

Hample, Dale. (2005) Arguing: exchanging reasons face to face. Mahwah, NJ: Lawrence Erlbaum.

Hample, Dale. (2003) Arguing skill. In Handbook of communication and social interaction skills. Mahwah, NJ: Lawrence Erlbaum, 439-478.

Hample, Dale, and Deepa Anagondahalli. (2015) Understandings of arguing in India and the United States: Argument frames, personalization of conflict, argumentativeness, and verbal aggressiveness. Journal of Intercultural Communication Research. 44 (1), 1-26.

Hample Dale, and Chrysi Rapanta. (2015) Orientations to interpersonal arguing in the United Arab Emirates, with comparisons to the United States, China, and India. Journal of Intercultural Communication Research, 44 (4),263-287.

Infante, Dominic. A. and Andrew S. Rancer. (1982) A conceptualization and measure of argumentativeness. Journal of Personality Assessment, 46, 72-80.

Infante, Dominic A. and Charles J. Wigley. (1986) Verbal aggressiveness: an interpersonal model and measure. Communication Monographs, 61-69.

Kant, Immanuel. (1999) Critique of Pure Reason. Cambridge: Cambridge University Press.

Khomenko, Iryna. (2018) Empiricalization as a Trend in Argumentation Study. Future Human Image. Volume 10, 20-28. https://doi.org/10.29202/fhi/10/2.

Khomenko, Iryna and Dale Hample. (2019) Comparative analysis of arguing in Ukraine and the USA. In Bart Garssen, David Godden, Gordon R., Mitchell, Jean H.M. Wagemans (Eds.) Proceedings of the Ninth Conference of the International Society for the Study of Argumentation. Amsterdam: Sic Sat, 628-639.

Lewiński, Marcin, Dale Hample, João Sàágua, and Mohammed Dima. (2018) Arguing in Portugal: A cross-cultural analysis. Journal of International and Intercultural Communication, 233253, https://doi.org/10.1080/17513057.2018.1450888

Perelman, Chaim and Lucy Olbrechts-Tyteca. (1958) Traité de l'argumentation: la nouvelle rhétorique. Paris: Presses Universitaires de France La Nouvelle Rhetorique.

Smeenk, Christopher and George Ellis. (2017) Philosophy of Cosmology. In Stanford Encyclopedia of Philosophy. https://plato.stanford.edu/entries/cosmology/

Toulmin, Stephen E. (2003) The uses of argument. Updated edn. Cambridge University Press.

Toulmin, Stephen E. (1958) The uses of argument. Cambridge University Press. 